\title{
Status of $\mathrm{H}$ I searches for CHVCs beyond the Local Group
}

\author{
R. Braun ${ }^{1}$ and W. B. Burton ${ }^{2}$ \\ 1 Netherlands Foundation for Research in Astronomy, PO Box 2, 7990 AA Dwingeloo, The Netherlands \\ 2 Sterrewacht Leiden, PO Box 9513, 2300 RA Leiden, The Netherlands
}

Received 3 April 2000 / Accepted 5 June 2001

\begin{abstract}
Growing evidence supports the suggestion that the compact high-velocity clouds of Hi (CHVCs) are located throughout the Local Group and continue to fuel galactic evolution. Recent distance estimates to individual objects lie in the range $150-850 \mathrm{kpc}$, implying an H I mass range of $10^{5.7} \rightarrow 10^{7.2} M_{\odot}$ together with sizes ranging from $2-12 \mathrm{kpc}$, while the average linewidth is $30 \mathrm{~km} \mathrm{~s}^{-1} F W H M$. It is natural to ask whether objects of this type would not already have been seen by current blind H I surveys of the extragalactic sky. We consider the properties of the deepest published surveys of this type and conclude that the sensitivity and coverage have now begun to reveal the high mass end of the distribution $\left(M_{\mathrm{HI}}>10^{7} M_{\odot}\right)$. Achieving detection limits an order of magnitude deeper should finally enable direct study of these systems beyond the Local Group, or definitively rule out their existence.
\end{abstract}

Key words. ISM: atoms - ISM: clouds - galaxy: evolution - galaxy: formation - galaxies: dwarf galaxies: Local Group

\section{Introduction}

Many aspects of the high-velocity cloud H I emission detected in large-area surveys at radial velocites between about -450 and $+400 \mathrm{~km} \mathrm{~s}^{-1}$ can be understood if this emission is associated with a population of low-mass, dark-matter-dominated structures distributed throughout the Local Group. This scenario was rejuvenated in a modern context by Blitz et al. (1999), based on the HVC tabulation of Wakker \& van Woerden (1991) and data of the Leiden/Dwingeloo H I Survey (LDS) of Hartmann \& Burton (1997), and by Braun \& Burton (1999), based on their extraction of compact, isolated HVCs from the LDS and confirmed with independent data. In the Local Group scenario, extended HVC complexes are nearby objects currently merging (or soon to merge) with the Galaxy, while the compact isolated high-velocity clouds (CHVCs) are the distant counterparts of the complexes which have as yet not strongly interacted with the massive Local Group constituents.

At least one of the HVC complexes (Chain A) lies at a distance between about 4 and $10 \mathrm{kpc}$ (van Woerden et al. 1999); another (Complex C) has a metallicity of only 0.09 solar (Wakker et al. 1999), implying an extragalactic origin even if the complex is currently nearby. If located

Send offprint requests to: R. Braun, e-mail: rbraun@nfra.nl at $D \sim 15 \mathrm{kpc}$, the $\mathrm{H}$ i mass of Complex $\mathrm{C}$ is $10^{7} M_{\odot}$ and its linear size is $15 \mathrm{kpc}$. Further evidence for the extragalactic nature of the CHVCs follows from the metallicity measurement of 0.05 solar for CHVC $125+41-207$ by Braun \& Burton (2000). The derived distance of this same object, which follows from a comparison of the $\mathrm{H}$ I column and volume densities, is $650 \pm 300 \mathrm{kpc}$. Comparably large distances and high dark-to-visible mass ratios (10 to 50) are also implied by the rotation signatures displayed by many CHVCs (Braun \& Burton 2000).

In the direct vicinity of massive galaxies we expect that these objects will live but shortly before tidal disruption and merger ensue. Such ongoing mergers are seen regularly in nearby galaxies, e.g. LMC (Luks \& Rohlfs 1992), M 33 (Deul \& van der Hulst 1987), M 101 (Kamphuis 1993), NGC 628 (Kamphuis \& Briggs 1992). Gas masses of a few times $10^{6}$ to more than $10^{8} M_{\odot}$ and physical dimensions of 10 to $20 \mathrm{kpc}$ are observed.

Counterparts to the CHVCs beyond the Local Group are more difficult to constrain. We argue here that current surveys are only beginning to achieve the appropriate combination of sensitivity and spatial coverage.

\section{CHVC populations, distance, mass and size}

Where and in what numbers might CHVC populations be expected to occur? Low-mass objects which have so far 
escaped assimilation by high-mass galaxies might be found in the vicinity of substantial, dynamically-young mass concentrations at distances less than the turnaround radius of the local over-density. In the case of the Local Group, this radius is at about 1.2 Mpc (Courteau $\&$ van den Bergh 1999) for a total mass of some $2.3 \times$ $10^{12} M_{\odot}$. The turn-around radius scales as the cube root of mass (Sandage 1986). For field galaxies with a typical luminosity of a few $10^{9} L_{\odot}$ and a mass of a few $10^{10} M_{\odot}$ the turn-around radius would lie at about $250 \mathrm{kpc}$. The total number of associated low-mass objects in a dynamically young system is expected to scale linearly with total mass (Klypin et al. 1999). Relative to the Local Group, we would then expect some 100 times fewer objects associated with a single $L^{*}$ galaxy in the field.

What is the typical H I mass, linewidth and linear size expected for a population of CHVCs? The average integrated flux of the 65 objects in the CHVC sample of Braun \& Burton (1999) is 100 with a dispersion of $75 \mathrm{Jy} \mathrm{km} \mathrm{s}^{-1}$; the average linewidth $30 \mathrm{kms}^{-1}$ FWHM; and the average $F W H M$ angular size is 50 with a dispersion of 25 arcmin when imaged with a 36 arcmin beam. Only a small number of distance estimates for CHVCs are currently in hand and even these are indirect, relying on an assumption about the typical thermal pressure of each source.

\subsection{CHVC distances utilizing compact opaque cores}

For objects that have compact opaque cores (in which the $\mathrm{H}$ I brightness temperature in emission is comparable to the gas kinetic temperature) with a column density enhancement, $d N_{\mathrm{HI}}$, of angular size, $\theta$, it is possible to estimate the distance from $D_{\mathrm{Core}}=d N_{\mathrm{HI}}^{\text {Core }} /\left(f n_{\mathrm{HI}} \theta\right)$, assuming crude spherical symmetry, if we have an estimate of the $\mathrm{HI}$ volume density, $n_{\mathrm{HI}}$ and its volume filling factor along the line-of-sight, $f$. Since the thermal pressure is given by $P=k_{\mathrm{B}} n_{\mathrm{HI}} T_{\mathrm{k}}$, we can rewrite the expression for distance as,

$$
\begin{aligned}
D_{\text {Core }}=\frac{670}{f} & \left(\frac{d N_{\mathrm{HI}}^{\text {Core }}}{10^{21} \mathrm{~cm}^{-2}}\right)\left(\frac{T_{\mathrm{k}}}{100 \mathrm{~K}}\right) \\
& \times\left(\frac{P / k}{100 \mathrm{~cm}^{-3} \mathrm{~K}}\right)^{-1}\left(\frac{\theta}{100^{\prime \prime}}\right)^{-1} \mathrm{kpc} .
\end{aligned}
$$

In general, only a crude lower limit to the distance follows from this approach since the filling factor is difficult to determine and might be substantially less than unity. However, in those cases where the core H I is essentially optically thick, a filling factor near unity can be plausibly assumed.

This method can perhaps best be demonstrated by using $\mathrm{M} 31$ as an example. The excess column density, $d N_{\mathrm{HI}}^{\text {Core }}=5 \pm 1 \times 10^{21} \mathrm{~cm}^{-2}$, and angular size, $\theta=60 \pm 20$ arcsec, of opaque H I clumps in the NorthEast half of M 31 can be estimated from Fig. 5 of Braun \& Walterbos (1992). The H I kinetic temperature, $T_{\mathrm{k}}=175 \pm 25 \mathrm{~K}$, and estimated thermal pressure, $P / k=1500 \pm 500 \mathrm{~cm}^{-3} \mathrm{~K}$, of the M 31 mid-disk are tabulated in Table 4 of the same reference. This yields the distance estimate: $D_{\mathrm{M} 31}=650 \pm 220 \mathrm{kpc}$, assuming a volume filling factor for these clumps of unity. While rather crude, this approach gives a plausible value for the distance to M 31.

Opaque $\mathrm{HI}$ cores have only been detected in one CHVC to date, namely CHVC125+41-207 (Braun \& Burton 2000), where an excess column density, $d N_{\mathrm{HI}}^{\text {Core }}=$ $1.0 \pm 0.2 \times 10^{21} \mathrm{~cm}^{-2}$, and angular size, $\theta=90 \pm 15$ arcsec, is measured in the H I clumps. A good estimate of the kinetic temperature follows from the measured linewidth in these clumps of only $2.0 \mathrm{~km} \mathrm{~s}^{-1} F W H M$, for which $T_{\mathrm{k}}=85 \pm 10 \mathrm{~K}$, while a peak brightness temperature of $75 \mathrm{~K}$ is observed, suggesting that a filling factor near unity might be considered.

What must still be estimated to employ Eq. (1) is the appropriate thermal pressure. Although the pressure, $P / k$, in the solar neighborhood of the Galaxy is well-determined at about $2000 \mathrm{~cm}^{-3} \mathrm{~K}$, it is expected to decline rapidly with distance from the Galactic plane (e.g. Wolfire et al. 1995) such that beyond about $10 \mathrm{kpc}$ we should encounter values, $P / k=100 \mathrm{~cm}^{-3} \mathrm{~K}$ or less due to the Galactic halo.

Within the inter-galactic medium (IGM) of the Local Group, it is difficult to determine what values of thermal pressure might be encountered. Current instrumentation has allowed diffuse X-ray emission to be detected from the IGM in several "poor" galaxy groups (e.g. Mulchaey et al. 1996). It must be noted that even the poorest of the currently detected groups is substantially richer than the Local Group and all have proven to be lacking in spirals. The X-ray detected systems have derived gas masses of about $10^{13} M_{\odot}$ (comparable to or less than the mass in galaxies), temperatures of about $1 \mathrm{keV}$ (corresponding to $10^{7} \mathrm{~K}$ ) and radii of about $500 \mathrm{kpc}$. Mulchaey et al. suggest that spiral-dominated galaxy groups are likely to have lower X-ray temperatures, of perhaps $0.2 \mathrm{keV}$. Assuming a correspondingly lower total gas mass of about $10^{12} M_{\odot}$ and radii between 500 and $1000 \mathrm{kpc}$ such as might apply to the Local Group, would imply a Local Group IGM thermal pressures in the range 160 to $20 \mathrm{~cm}^{-3} \mathrm{~K}$.

Braun \& Burton (2000) have considered the total pressure implied by assuming hydrostatic equilibrium of a selfgravitating gaseous disk. The self-gravity of the WNM in a diffuse disk system accounts for a contribution, $P / k=$ $40\left(N_{\mathrm{HI}}^{\text {Halo }}(0) / 10^{20} \mathrm{~cm}^{-2}\right)^{2} \mathrm{~cm}^{-3} \mathrm{~K}$ in the absence of a significant dark matter contribution to the disk mass. The peak column densities of WNM found in CHVCs are in the range $10^{19.5}-10^{20} \mathrm{~cm}^{-2}$ (Burton et al. 2001).

The resulting thermal pressure, the sum of selfgravitating and external components, at the interface of the warm neutral halo with the cool condensed cores, of about $P / k=100 \pm 50 \mathrm{~cm}^{-3} \mathrm{~K}$ is in very good agreement with that expected theoretically (Wolfire et al. 1995). This transition pressure is found to apply over a wide range of physical conditions (metal abundance, radiation field and dust content). Taking the value, $P / k=100 \pm 50 \mathrm{~cm}^{-3} \mathrm{~K}$, allows calculation of the distance to CHVC125+41-207, yielding $D_{\mathrm{C} 125}=600 \pm 300 \mathrm{kpc}$. 


\subsection{CHVC distances utilizing edge profiles}

A second method of distance estimation (Burton et al. 2001) makes use of the column density profiles of the diffuse WNM halos of these objects. These halos can be welldescribed by the sky-plane projection of a spherical exponential distribution of the H I volume density,

$n_{\mathrm{HI}}(r)=n_{\mathrm{o}} \mathrm{e}^{-r / h}$

in terms of the radial distance, $r$, and exponential scale length, $h$. The projected column density distribution is then,

$N_{\mathrm{HI}}^{\text {Halo }}(r)=2 h n_{\mathrm{O}}\left[\frac{r}{h} K_{1}\left(\frac{r}{h}\right)\right]$

where $K_{1}$ is the modified Bessel function of order 1 . In this formulation, the peak halo column density is given simply by,

$N_{\mathrm{HI}}^{\mathrm{Halo}}(0)=2 h n_{\mathrm{o}}$.

The distance of the source can then be calculated from,

$D_{\text {Halo }}=\frac{N_{\mathrm{HI}}^{\text {Halo }}(0)}{2 h n_{\mathrm{o}}}=\frac{N_{\mathrm{HI}}^{\text {Halo }}(0) k_{\mathrm{B}} T_{\mathrm{k}}^{\text {Halo }}}{2 h P}$

where the implicit assumption has been made of a unit filling factor for the halo gas. If we assign the measured temperature of $T_{\mathrm{k}}^{\text {Halo }}=10^{4} \mathrm{~K}$ to the halo gas this becomes,

$$
\begin{aligned}
D_{\text {Halo }}= & 335\left(\frac{N_{\mathrm{HI}}^{\mathrm{Halo}}(0)}{10^{19} \mathrm{~cm}^{-2}}\right) \\
& \times\left(\frac{P / k}{100 \mathrm{~cm}^{-3} \mathrm{~K}}\right)^{-1}\left(\frac{h}{100^{\prime \prime}}\right)^{-1} \mathrm{kpc} .
\end{aligned}
$$

The peak halo column densities, $N_{\mathrm{HI}}^{\mathrm{Halo}}(0)$, and scalelengths, $h$, can be determined from sensitive total power observations, like the deep Arecibo cross-cuts obtained for a sample of ten objects by Burton et al. (2001). Assuming, as before, a nominal thermal pressure of $P / k=$ $100 \pm 50 \mathrm{~cm}^{-3} \mathrm{~K}$ at the interface of the cool and warm neutral H I, yields distance estimates which range from 150 to $850 \mathrm{kpc}$ for the various objects studied.

An independent method of distance estimation (Burton et al. 2001) follows from simply equating the angular H I scale-lengths observed in the CHVCs with the mean physcial scale-length, $h=1.1 \pm 0.4 \mathrm{kpc}$, measured in a sample of low mass dwarf galaxies. This yields distances in the range 320 to $730 \mathrm{kpc}$.

Apparently, a wide range of distances, spanning almost an order of magnitude, is indicated for the Local Group CHVC population. If a distance range of $150 \rightarrow 850 \mathrm{kpc}$ is representative for the $\mathrm{CHVCs}$, then the corresponding $\mathrm{H}$ I mass range is $M_{\mathrm{HI}}=10^{5.7} \rightarrow 10^{7.2} M_{\odot}$. The distribution of masses over this range is not yet known, but is likely to be more heavily populated at the low end than the high. The critical H I mass detection threshold is therefore about $10^{6} M_{\odot}$, if a large fraction of the population is to be detected. If the typical radial profile of the CHVCs were Gaussian, then the corresponding range of FWHM linear sizes would be $2 \rightarrow 12 \mathrm{kpc}$. Since the edge profiles are instead observed to be exponential, these objects will appear slightly resolved when observed with any telescope beam broader than that of the high column density cores of about 5-10 arcmin (Braun \& Burton 2000), corresponding to 1-2 kpc. Unfortunately, with an exponential edge profile, the observed object "size" and "total mass" will depend not only on the resolution used, but also on the sensitivity achieved. For a 2-D circular exponential distribution, the fractional flux contained within a radius of one, two and three scale-lengths is 26,59 and $80 \%$. The implication is that only for beam sizes greater than about $20 \mathrm{kpc}$, is it likely that the entire flux of an object will be detected in cases of modest signal-to-noise ratio.

\section{Synthesis surveys}

One method of achieving "blind" survey coverage for low mass objects is to utilize the relatively large field-of-view of an interferometeric array when observing the $\mathrm{H}$ I distribution in nearby galaxies to obtain serendipitous detection of nearby uncataloged systems. Some aspects of this problem have been considered previously by Blitz et al. (1999). With a typical interferometric field of view of 30 arcmin, an area with linear size of $90 D_{10} \mathrm{kpc}$ (normalized to a source at $10 \mathrm{Mpc}$ ) is probed with a single pointing. Since correlator capacity is often a limiting factor for such observations, the typical velocity coverage obtained is often only about 1.5 times the velocity extent of the target galaxy. Depending on the goals of a particular observing program, this entire field and velocity coverage may not even be analyzed; but if it were, the probability of intercepting an associated object would be proportional to the total number of objects, $N_{\text {Grp }}$ for a galaxy group or $N_{\text {Gal }}$ for an isolated galaxy, as well as the fractional area and velocity extent observed. Assuming that the velocity coverage is sufficient to sample the entire associated population, this corresponds to $N=0.002 N_{\mathrm{Grp}} D_{10}^{2}$ for a distribution extending out to $1 \mathrm{Mpc}$ (a distance comparable to the Local Group turn-around radius) or $N=0.03 N_{\text {Gal }} D_{10}^{2}$ for an isolated galaxy. Only for values of $N_{\text {Grp }}$ as large as 500 or $N_{\mathrm{Gal}}=30$ would one expect serendipitous detections in individual pointings that achieve a detected mass sensitivity of better than about $10^{6} M_{\odot}$ over $30 \mathrm{~km} \mathrm{~s}^{-1}$ with a beam size larger than about $20 \mathrm{kpc}$.

The required mass sensitivity for CHVC detection in external galaxy groups has not yet been generally attained. Taylor et al. $(1995,1996)$ have searched the fields of 21 apparently isolated H II galaxies and of 17 low surface brightness dwarf galaxies. The targeted galaxies were at a typical distance of about $25 \mathrm{Mpc}$, where the half-power field-of-view is $225 \mathrm{kpc}$ and the spatial resolution about $8 \mathrm{kpc}$. The observations reached an average five sigma mass sensitivity over $30 \mathrm{~km} \mathrm{~s}^{-1}$ of about $2.5 \times 10^{7} M_{\odot}$ at the field center in the case of the HII galaxies and $1.7 \times 10^{7} M_{\odot}$ in the case of the LSB dwarf galaxies. While a total of about 17 companion galaxies were discovered 
in this survey, all but one (UM422C) were found to have optical counterparts detectable at surface brightnesses of $23 \mathrm{mag} \operatorname{arcsec}^{-2}$. Since the H I detection thresholds have very little overlap with the mass range of the Local Group CHVC population this result can be readily understood.

Another sensitive search for H I emission was carried out by Van Gorkom et al. (1993) in the vicinity of Ly $\alpha$ clouds in a single 30' FWHM field centered on 3C 273 and covering +840 to $+1840 \mathrm{~km} \mathrm{~s}^{-1}$. The field is located some $10^{\circ}$ (or about $2.6 \mathrm{Mpc}$ ) from the center of the Virgo Cluster (at an assumed distance of $15 \mathrm{Mpc}$ ). The rms noise in the center of their field corresponded to a $5 \sigma$ mass sensitivity of $2.5-4.5 \times 10^{6} M_{\odot}$, in a single $41.6 \mathrm{~km} \mathrm{~s}^{-1}$ channel at linear resolutions of 1.5-4.2 kpc. Van Gorkom et al. estimate a $5 \sigma$ mass sensitivity at $10 \mathrm{kpc}$ resolution (to better match the expected CHVC size) of $1.1 \times 10^{7} M_{\odot}$ at the field center. Spatially averaged over the $30^{\prime}$ FWHM Gaussian field-of-view yields a $5 \sigma$ average mass limit of $1.6 \times 10^{7} M_{\odot}$ over a region $0.013 \mathrm{Mpc}^{2}$ in extent. The fractional area sampled relative to the impact parameter of $2.6 \mathrm{Mpc}$ is $0.06 \%$. No H I emission was detected, implying an upper limit of some 2000 uniformly distributed objects with $M_{\mathrm{HI}}>1.6 \times 10^{7} M_{\odot}$, if the imaged field is representative. We note that the Virgo Cluster is a dynamically evolved system, for which the long term survival of a low mass population is uncertain. But, in any case, the average mass sensitivity of this search is insufficient to detect large numbers of CHVC counterparts.

More recently Pisano \& Wilcots $(1999,2000)$ have surveyed the fields of 34 isolated galaxies with distances in the range 21 to $45 \mathrm{Mpc}$. With a linear resolution of $6-13 \mathrm{kpc}$, they achieve a $5 \sigma$ mass sensitivity over $30 \mathrm{~km} \mathrm{~s}^{-1}$ of $1.4 \pm 0.6 \times 10^{7} M_{\odot}$ at each field center. This sensitivity begins to overlap with the range of expected CHVC masses near the field center. However, averaged over the primary beam out to the half power point, corresponding to radii of $90-190 \mathrm{kpc}$, the mass sensitivity is decreased to $2 \pm 1 \times 10^{7} M_{\odot}$, where effectively no detections are expected. Although several gas-rich companions are detected in this survey, all but possibly one are accompanied by optical counterparts.

\section{Total power surveys}

One of the earliest systematic searches for H I in galaxy groups was that of Lo \& Sargent (1979). Large regions of the sky in the vicinity of three nearby galaxy groups were searched using the OVRO $40 \mathrm{~m}$ telescope for H I emission features without obvious optical counterparts. The $5 \sigma$ mass sensitivity for a $35 \mathrm{~km} \mathrm{~s}^{-1} F W H M$ linewidth was $4 \times 10^{7} M_{\odot}$ for a region of 132 square degrees (or $0.4 \mathrm{Mpc}^{2}$ ) adjacent to $\mathrm{M} 81$, while mass limits of $8 \times 10^{7}$ over $0.7 \mathrm{Mpc}^{2}$ and $46 \times 10^{7} M_{\odot}$ over $5.7 \mathrm{Mpc}^{2}$ were obtained in 90 square degree regions near the $\mathrm{CVnI}$ group and NGC 1023 respectively. A total of six gas-rich objects were subsequently detected in follow-up work using the $100 \mathrm{~m}$ Effelsberg telescope, although all of them do have (faint) optical counterparts. Comparison with our mass estimates above indicates that the non-detection of an extensive CHVC population is entirely in keeping with the likely mass distribution of Local Group CHVCs.

\subsection{The Arecibo Hı strip survey}

A sensitive, large area Hi survey has been the Arecibo H I Strip Survey (AHISS) by Zwaan et al. (1997). In fact, Zwaan \& Briggs (2000) claim that the AHISS would have made numerous detections of CHVCs if they did populate the outer environments of galaxies and galaxy groups. In order to critically assess this claim, we have carefully considered the attributes of the survey which are welldocumented by Sorar (1994).

The spatial coverage of the AHISS consists of two repeatedly-observed strips, one covering the right ascension interval $18^{\mathrm{h}}$ to $05^{\mathrm{h}}$ at $\delta=14^{\circ} 14^{\prime}$, and the other covering $01^{\mathrm{h}}$ to $10^{\mathrm{h}} .7$ at $\delta=23^{\circ} 09^{\prime}$. The velocity coverage extends from -700 to $+7500 \mathrm{~km} \mathrm{~s}^{-1}$. The noise level of the AHISS data depends on position as well as velocity for a number of reasons. Firstly, varying integration times were obtained for different positions. Secondly, the receiver gain varied significantly with frequency. For the $\delta=14^{\circ} 14^{\prime}$ strip this implied a factor of two degraded sensitivity at $0 \mathrm{~km} \mathrm{~s}^{-1}$ than at $+5000 \mathrm{~km} \mathrm{~s}^{-1}$, while for the $\delta=23^{\circ} 09^{\prime}$ strip, the degradation at $0 \mathrm{~km} \mathrm{~s}^{-1}$ was limited to about $15 \%$. And lastly, confusion and spectral baseline residuals due to bright, extended emission from the Galaxy preclude detection of objects within about $\left|V_{\mathrm{GSR}}\right|<200 \mathrm{~km} \mathrm{~s}^{-1}$.

The average rms level of the $\delta=14^{\circ}$ strip is $0.95 \mathrm{mJy}$ beam $^{-1}$ at $16 \mathrm{~km} \mathrm{~s}^{-1}$ resolution at velocities near $+5000 \mathrm{~km} \mathrm{~s}^{-1}$, rising to 1.9 mJy beam ${ }^{-1}$ at low recession velocites. For the $\delta=23^{\circ}$ strip, the average noise level is $0.75 \mathrm{mJy}$ beam $^{-1}$ at $16 \mathrm{~km} \mathrm{~s}^{-1}$ resolution between about +5000 and $+1000 \mathrm{~km} \mathrm{~s}^{-1}$, rising to $0.85 \mathrm{mJy}$ beam $^{-1}$ at low recession velocities.

The spatial coverage of the AHISS is given by the track of the telescope beam (about $180 \operatorname{arcsec} F W H M$ at $1400 \mathrm{MHz}$ ) along the indicated right ascension intervals. The maximum sensitivity, noted above, is only obtained for completely unresolved sources which pass within the central 20 arcsec or so of the beam response. If instead we consider a realistic search area comparable in width to the beam $F W H M$, then the average detected flux is down by a factor of 0.81 for an approximately Gaussian main lobe. This diminished response must be taken into account when assessing the sensitivity for unresolved sources over the full 180 arcsec width. If instead, a source is significantly resolved by the survey beam, then the fraction of detected flux at a given offset position is given by the convolution of the beam and source shapes. In the case of two circular Gaussians of similar FWHM, with random linear offsets in the range $\pm H W H M$, the average detected flux is reduced by a factor of 0.64 from the intrinsic flux.

The mass sensitivity of the AHISS averaged over the $F W H M$ of the telescope beam can now be assessed as 
a function of recession velocity. For nearby systems, say at $5 \mathrm{Mpc}$ distance, the telescope beam corresponds to only $4.4 \mathrm{kpc}$ and any detected system will be strongly resolved. The rms noise averaged over the $\delta=14^{\circ}$ and $23^{\circ}$ strips at $V_{\mathrm{GSR}}=300 \mathrm{~km} \mathrm{~s}^{-1}$, is $1.4 \mathrm{mJy}_{\text {beam }}{ }^{-1}$ over $16 \mathrm{~km} \mathrm{~s}^{-1}$, yielding a $5 \sigma$ limit on detected signal strength of $160 \mathrm{mJy}$ beam ${ }^{-1}-\mathrm{km} \mathrm{s}^{-1}$ over the typical $32 \mathrm{~km} \mathrm{~s}^{-1}$ linewidth. The average intrinsic signal strength that gives this response will be at least a factor of $1 / 0.64$ higher (due to offsets from the beam center) yielding a $5 \sigma$ mass limit of $1.5 \times 10^{6} M_{\odot}$. At this distance the AHISS is indeed capable of detecting a large part of the expected CHVC mass distribution, even with some further reduction in detected flux due to the exponential edge profiles.

At a distance of $15 \mathrm{Mpc}$, the telescope beam coresponds to $13 \mathrm{kpc}$, which is comparable to the expected source size. The rms noise averaged over the $\delta=14^{\circ}$ and $23^{\circ}$ strips at $V_{\mathrm{GSR}}=1000 \mathrm{~km} \mathrm{~s}^{-1}$, is $1.1 \mathrm{mJy}$ beam ${ }^{-1}$ over $16 \mathrm{~km} \mathrm{~s}^{-1}$, yielding a $5 \sigma$ limit on detected signal strength of $125 \mathrm{mJy}$ beam ${ }^{-1}-\mathrm{km} \mathrm{s}^{-1}$ over the typical $32 \mathrm{~km} \mathrm{~s}^{-1}$ linewidth. The average intrinsic signal strength will again be higher by the beam averaging factor of $1 / 0.64$ (for the comparable beam and source size case), yielding a $5 \sigma$ mass limit of $1.0 \times 10^{7} M_{\odot}$. This constitutes a practical distance limit to the AHISS for expected detection of the upper mass end of the CHVC mass distribution.

We searched the Garcia (1993) catalog of galaxy groups for all those in the range +200 to $+1200 \mathrm{~km} \mathrm{~s}^{-1}$ and within $1 \mathrm{Mpc}$ projected distance of the AHISS coverage. Only one cataloged galaxy group is intercepted by the AHISS in this range, namely the NGC 628 group centered at $(\alpha, \delta, V)=\left(01^{\mathrm{h}} 36^{\mathrm{m}}, 14^{\circ} 55^{\prime},+570 \mathrm{~km} \mathrm{~s}^{-1}\right)$, at a distance of about $10 \mathrm{Mpc}$. There is no other instance of the AHISS coverage passing near a group of galaxies within $15 \mathrm{Mpc}$.

The AHISS sensitivity in the NGC628 region is $1.4 \mathrm{mJy}_{\text {beam }}{ }^{-1}$ over $16 \mathrm{~km} \mathrm{~s}^{-1}$, while the telescope beam corresponds to about $9 \mathrm{kpc}$. With a beam averaging factor of $1 / 0.64$, this yields a $5 \sigma$ mass sensitivity of $5.7 \times 10^{6} M_{\odot}$ over the expected linewidth of $32 \mathrm{~km} \mathrm{~s}^{-1}$. This sensitivity should be sufficient to allow detection of the more massive members of a CHVC population, if they happened to lie within the survey strip. The AHISS coverage passes within $120 \mathrm{kpc}$ of the nominal NGC 628 group center, so the group area intercepted by this strip corresponds to a region about $9 \mathrm{kpc}$ wide (for the 180 arcsec beam $F W H M$ at $D=10 \mathrm{Mpc}$ ) and some $2 \mathrm{Mpc}$ long. This area corresponds to some $0.6 \%$ of the total group area $\left(\pi R^{2}\right)$ of about $3.1 \mathrm{Mpc}^{2}$. For a uniform distribution of objects, the AHISS non-detection implies that the total number of such objects exceeding $5.7 \times 10^{6} M_{\odot}$ should be less than about 300 .

The next nearest group covered by the AHISS survey is the NGC 3227 group at $(\alpha, \delta, V)=$ $\left(10^{\mathrm{h}} 19^{\mathrm{m}}, 21^{\circ} 23^{\prime},+1250 \mathrm{~km} \mathrm{~s}^{-1}\right)$. Since this is covered in the $\delta=23^{\circ}$ strip at a velocity greater than $+1000 \mathrm{~km} \mathrm{~s}^{-1}$, the flux sensitivity is 0.75 mJy beam ${ }^{-1}$ at $16 \mathrm{~km} \mathrm{~s}^{-1}$ resolution. The $5 \sigma$ mass sensitivity at an estimated distance of $19.2 \mathrm{Mpc}$ is then $M_{\mathrm{HI}}=1.1 \times 10^{7} M_{\odot}$ over
$32 \mathrm{~km} \mathrm{~s}^{-1}$. This sensitivity is sufficient to detect only the most massive objects in a CHVC population. Since the survey coverage passes through the group at an impact parameter of about $590 \mathrm{kpc}$, the $17 \mathrm{kpc}$ beamwidth samples a fractional group area of about $0.9 \%$. The AHISS non-detection implies that the number of objects exceeding $1.1 \times 10^{7} M_{\odot}$ should be less than about 200 .

We have also searched the LEDA database for all cataloged galaxies with recession velocities (corrected for Virgo-centric infall) in the range +200 to $+1300 \mathrm{~km} \mathrm{~s}^{-1}$ and projected separations of less than $250 \mathrm{kpc}$ from the AHISS strip coverage. Apart from galaxies belonging to the NGC 628 group, and therefore already considered above, a total of 7 other galaxies lie this close to the AHISS coverage. Six of the seven are low mass dwarf irregular galaxies (PGC 86669, 169957, 169968, 169969, and UGC $1561,2905)$ with $\mathrm{H}$ I line widths in the range $30-60 \mathrm{~km} \mathrm{~s}^{-1}$ FWHM. Three of these sources were in fact discovered in the "Arecibo Slice" survey discussed below. The seventh is a low luminosity spiral (UGC 5672, $M_{\mathrm{B}}=-15.4$, $\left.M_{\mathrm{HI}}=5 \times 10^{7} M_{\odot}\right)$. None of these galaxies appear to be fruitful starting points for a search for associated low-mass satellite systems, since even the most massive is only about $0.01 L^{*}$. Since turn-around radius scales as the cube root of total mass we expect turn-around radii of $55 \mathrm{kpc}$ at most for these systems, rather than the nominal value of $250 \mathrm{kpc}$ for an $L^{*}$ galaxy. Exactly one of the dwarf irregulars lies within $55 \mathrm{kpc}$ of the AHISS track, PGC 169968. At a distance of $8 \mathrm{Mpc}$, the AHISS $5 \sigma$ mass limit is $2.2 \times 10^{6} M_{\odot}$ over $32 \mathrm{~km} \mathrm{~s}^{-1}$ and the $F W H M$ beam is some $7 \mathrm{kpc}$ broad. At an impact parameter of $17 \mathrm{kpc}$ from an assumed $55 \mathrm{kpc}$ radius distribution, the strip samples a region of length $100 \mathrm{kpc}$. The fractional area sampled by the AHISS coverage is then $18 \%$. The absence of detected companions for this object in the AHISS implies that its CHVC population should number less than about 10 for objects with mass greater than $2.2 \times 10^{6} M_{\odot}$.

\subsection{The "Arecibo Slice" survey}

Another recent H I survey is that of Spitzak \& Schneider (1998) who utilized the Arecibo telescope to survey the region between right ascension $22^{\mathrm{h}}$ to $03^{\mathrm{h}} 24^{\mathrm{m}}$ and $\delta=22^{\circ} 58^{\prime}$ to $\delta=23^{\circ} 47^{\prime}$. The flux sensitivity at the hexagonally spaced sampling points was typically $1.7 \mathrm{mJy}$ beam ${ }^{-1}$ over $16 \mathrm{~km} \mathrm{~s}^{-1}$ at $V_{\mathrm{Hel}}=5300 \mathrm{~km} \mathrm{~s}^{-1}$, decreasing to $81 \%$ of this value at $8340 \mathrm{~km} \mathrm{~s}^{-1}$ and to $56 \%$ at $100 \mathrm{~km} \mathrm{~s}^{-1}$. Since the sampling points were spaced quite coarsely relative to the beam size (4.1 arcmin spacing and a 3.3 arcmin beam), the flux sensitivity is estimated to vary by about a factor of three, depending on whether sources were located at sampling points or exactly between them. The corresponding average $5 \sigma$ mass sensitivity at a distance of $5 \mathrm{Mpc}$ is about $4 \times 10^{6} M_{\odot}$, increasing to $1.5 \times 10^{7} M_{\odot}$ at $10 \mathrm{Mpc}$. We searched for galaxy groups in the Garcia (1993) catalog in the velocity range +200 to $+1200 \mathrm{~km} \mathrm{~s}^{-1}$ within a projected seperation of $1 \mathrm{Mpc}$ of the survey coverage, 
but found none. The only four LEDA cataloged galaxies in this velocity range which lie inside the survey coverage or within a projected turn-around radius are PGC 169957, 169968, 169969 and UGC 1561. These are all very low mass systems as already noted above in connection with the AHISS survey discussion, where the same galaxies were also encountered. The first three of these objects were discovered in the "Arecibo Slice" survey. Faint optical counterparts are seen for the PGC 169968 and 169957, which have $\mathrm{H}$ I masses of about 1.3 and $3 \times 10^{7} M_{\odot}$ respectively. PGC 169969 is the lowest H I mass object found, in this and any other blind $\mathrm{HI}$ survey to date, of only about $8 \times 10^{6} M_{\odot}$. No optical counterpart to this source has yet been detected, although a relatively bright fore-ground star makes this a difficult undertaking. These objects fit into the more general trend implied by Fig. 9 of Spitzak \& Schneider (1998) for H I selected objects to be increasingly gas-dominated at low galaxy mass.

\subsection{HIPASS survey of the Cen A galaxy group}

A portion of the Cen A galaxy group has recently been subjected to a similar search by Banks et al. (1999) using the HIPASS data obtained with the Parkes $64 \mathrm{~m}$ telescope. They searched an area of 600 square degrees, corresponding to about $2.2 \mathrm{Mpc}^{2}$, for $\mathrm{H}$ I detections in the velocity range $V_{\mathrm{Hel}}=200-1000 \mathrm{~km} \mathrm{~s}^{-1}$. The $5 \sigma$ mass sensitivity over $35 \mathrm{~km} \mathrm{~s}^{-1}$ was $7 \times 10^{6} M_{\odot}$ for a nominal distance of $3.5 \mathrm{Mpc}$, while the 15 arcmin angular resolution corresponds to $15 \mathrm{kpc}$. This mass sensitivity has substantial overlap with the mass distribution we derive for the Local Group CHVC population, although a modest loss in total flux might be expected in the $15 \mathrm{kpc}$ beam. The Cen A group has six major members within a total extent of about $1.9 \mathrm{Mpc}$, making it about twice as rich as the Local Group. Banks et al. detect 10 new group members in their survey area, most of which have $M_{\mathrm{HI}}$ near the $\sim 10^{7} M_{\odot}$ sensitivity limit. Five of these were not previously cataloged, despite deep optical searches in this region, and have extremely low optical luminosities (below $\left.M_{\mathrm{B}} \sim-15\right)$ and surface brightness $\left(<\mu_{\mathrm{B}}>\sim 26 \mathrm{mag}\right.$ $\left.\operatorname{arcsec}^{2}\right)$. These five objects have an average H I mass of $2.0 \pm 0.8 \times 10^{7} M_{\odot}$ and a $F W H M$ linewidth of $34 \pm 4 \mathrm{~km} \mathrm{~s}^{-1}$. While this is still at the high mass end of the Local Group CHVC distribution, it is possible that a new population of low mass, extremely star-poor systems is starting to emerge. An even more extreme object in this class seems to the possible Cen A group member HIPASS J1712-64 discovered by Kilborn et al. (2000). With $V_{\mathrm{Hel}}=460 \mathrm{~km} \mathrm{~s}^{-1}$ it is well-removed in velocity from any confusing emission and in agreement with that of the group. At an estimated distance of $3.2 \mathrm{Mpc}$, it has an $\mathrm{HI}$ mass of $1.7 \times 10^{7} M_{\odot}, 15 \mathrm{kpc}$ size (at $10^{19} \mathrm{~cm}^{-2}$ column density) and lies at a projected seperation of $1.1 \mathrm{Mpc}$ from the massive Circinus galaxy. No optical counterpart to this object has been detected down to a limiting surface brightness of $\mu_{\mathrm{B}} \sim 27$ mag $\operatorname{arcsec}^{2}$.

\subsection{Targeted survey of groups}

A targeted survey of galaxy group environments was recently carried out by Zwaan (2001). A sparsely sampled grid of 60 pointings was observed with the Arecibo telescope centered on each of five galaxy groups at recession velocities of 1770, 1870, 2280, 2910 and $3000 \mathrm{~km} \mathrm{~s}^{-1}$ (corresponding to distances between about 27 and $46 \mathrm{Mpc}$ ). The 60 pointings were distributed over rectangular areas of about $2.5 \times 1.5 \mathrm{Mpc}$. At these distances each $F W H M$ beam has an area of 24-40 kpc. Together, each grid of 60 pointings covered an area varying from $0.027-$ $0.075 \mathrm{Mpc}^{2}$, corresponding to a fractional coverage of between $0.7-2.0 \%$ for the various groups sampled. An average flux sensitivity of $0.75 \mathrm{mJy}$ beam $^{-1}$ over $10 \mathrm{~km} \mathrm{~s}^{-1}$ was obtained, corresponding to $5 \sigma$ mass limits $1.1-3.2 \times$ $10^{7} M_{\odot}$ over $30 \mathrm{~km} \mathrm{~s}^{-1}$ at each beam center. Since the survey area is sparsely sampled we must consider the sensitivity averaged over the beam area, rather than simply taking the peak value. The average $5 \sigma$ mass sensitivity over each beam $F W H M$ then varies between $1.6-4.6 \times 10^{7} M_{\odot}$ over $30 \mathrm{~km} \mathrm{~s}^{-1}$. Only in the case of the nearest two galaxy groups is there a small degree of overlap of the achieved mass sensitivity with the expected CHVC mass distribution. The constraint that follows from non-detections over $0.75 \%$ of the group area, is that there must be less than about 100 objects with $\mathrm{H}$ I mass exceeding $1.7 \times 10^{7} M_{\odot}$ associated with the NGC 5798 and NGC 5962 galaxy groups. Unfortunately this is not a very strong constraint.

\section{Comparison with other work}

Zwaan \& Briggs (2000) discuss detection statistics of primordial gas clouds near galaxies and galaxy groups based on the AHISS data. They state that the survey "... probed the haloes of $\sim 300$ cataloged galaxies and the environments of $\sim 14$ groups with sensitivity to neutral hydrogen masses $\geq 10^{7} M_{\odot}$ ". As we have shown, a limiting H I mass of $10^{7} M_{\odot}$ is indeed a minimal requirement for the expected detection of such features, and even this will only allow detection of the most massive objects in the expected distribution. The AHISS survey attributes (Sorar 1994) lead to a distance limit of $15 \mathrm{Mpc}$ for the $5 \sigma$ detection of an $\mathrm{HI}$ mass of $10^{7} M_{\odot}$ within the $15 \mathrm{kpc}$ Arecibo beam over the expected linewidth of $32 \mathrm{~km} \mathrm{~s}^{-1}$. Out to this distance the AHISS survey coverage passes within the turn-around radius of exactly one galaxy group (the NGC 628 group) and one cataloged galaxy (PGC 169968). Relaxing this distance limit to $20 \mathrm{Mpc}$, brings only one additional galaxy group (the NGC 3227 group) and no additional galaxies into range.

There are several reasons for this apparent discrepancy. The single most important factor is the assumed distance, and hence mass, of the population being sought. Assuming a baryon-to-dark-mass fraction, $f=0.1$, and calculating a stability distance for the entire Wakker \& van Woerden (1991) HVC sample following Blitz et al. (1999) leads to a typical distance of $1 \mathrm{Mpc}$ and H I masses 
in the range $10^{7}-10^{8.5} M_{\odot}$. We have shown above that current distance estimates to individual CHVCs in the Local Group lie in the range $150-850 \mathrm{kpc}$ and that the corresponding $\mathrm{H}$ I mass range for CHVCs cataloged in BB99 is $M_{\mathrm{HI}}=10^{5.7} \rightarrow 10^{7.2} M_{\odot}$.

Zwaan \& Briggs also assume a nominal $5 \sigma$ column density limit of $10^{18} \mathrm{~cm}^{-2}$, corresponding to a flux density rms of $0.75 \mathrm{mJy}$ beam ${ }^{-1}$ over $16 \mathrm{~km} \mathrm{~s}^{-1}$ that applies uniformly to a 180 arcsec wide strip, in assessing the survey detectability of an extragalactic CHVC population. The AHISS flux density rms is in fact typically higher than $0.75 \mathrm{mJy}^{\text {beam }}{ }^{-1}$ over $16 \mathrm{~km} \mathrm{~s}^{-1}$ as discussed specifically above, especially at low velocities. In addition, the assumed linewidth of $16 \mathrm{~km} \mathrm{~s}^{-1}$ is about a factor of two lower than the measured linewidths of this class of object, making actual detection more difficult by a factor of $\sqrt{2}$. Furthermore, the average detected response across the 180 arcsec survey strip is reduced by about $50 \%$ for sources which are comparable in size to the beam. Together these factors degrade the effective detection sensitivity by more than a factor of three.

And finally, Zwaan \& Briggs have assumed that each cataloged field galaxy, independant of mass, might host a comparably large population of associated satellites extending out to a radius of $1 \mathrm{Mpc}$. In fact, it is reasonable to expect (e.g. Klypin et al. 1999) that the number of associated low mass satellites might be proportional to the host mass and that the radius over which they might extend be the turn-around radius of the host mass (which scales as $M^{1 / 3}$ ).

\section{Conclusions}

Recent distance estimates for a sample of ten compact high-velocity clouds (CHVCs) yield values in the range 150 to $850 \mathrm{kpc}$ (Burton et al. 2001). The corresponding $\mathrm{H}$ I masses of the BB99 catalog of CHVCs range between $10^{5.7} \rightarrow 10^{7.2} M_{\odot}$ over an average linewidth of $30 \mathrm{~km} \mathrm{~s}^{-1}$. Interferometric surveys of isolated fields have not generally achieved the combination of sensitivity and sky coverage to put strong constraints on CHVC populations in galaxy groups. The searches for low mass companions reported earlier by Taylor et al. $(1995,1996)$ and more recently by Pisano \& Wilcots $(1999,2000)$ fall short of probing the expected CHVC mass range.

The most sensitive large-area H I surveys are just beginning to sample the upper mass end of the implied CHVC mass distribution. The AHISS survey (Zwaan et al. 1997) could have detected objects with $M_{\mathrm{HI}}=10^{7} M_{\odot}$ out to a maximum distance of 15 Mpc. Unfortunately, out to this distance, the AHISS sky coverage passes within the turn-around radius of only a single galaxy group and a single isolated galaxy. The AHISS nondetections allow placing an upper limit of about $300 \mathrm{ob}-$ jects exceeding $M_{\mathrm{HI}}=5.7 \times 10^{6} M_{\odot}$ within a radius of $1 \mathrm{Mpc}$ of the NGC 628 galaxy group. A targeted search of the environments of several galaxy groups (Zwaan 2001) also falls short of reaching the required mass sensitivity.
The Arecibo Slice survey (Spitzak \& Schneider 1998) has detected several objects which overlap in $\mathrm{H}$ I mass with the CHVCs. The least massive of these $\left(M_{\mathrm{HI}} \sim 8 \times 10^{6} M_{\odot}\right)$ has no detected optical counterpart, in keeping with a more general trend for low mass systems to be increasingly gas-dominated.

The HIPASS survey has allowed detection of a small number of low mass galaxies in the Cen A galaxy group with extremely low surface brightness optical counterparts $\left(<\mu_{\mathrm{B}}>\sim 26 \mathrm{mag} \operatorname{arcsec}^{2}\right)$. This was accomplished with a $5 \sigma$ H I mass sensitivity of about $7 \times 10^{6} M_{\odot}$ (Banks et al. 1999). In addition, at least one object has been found with no detected optical counterpart down to $\mu_{\mathrm{B}} \sim 27 \mathrm{mag} \operatorname{arcsec}^{2}$ (Kilborn et al. 2000). These objects appear to be the highest mass counterparts of the Local Group CHVC population.

If the estimated distances of the Local Group CHVC population discussed in Sect. 2 are correct then pushing the detection limits down by another order of magnitude, to $M_{\mathrm{HI}}=10^{6} M_{\odot}$ (over $15 \mathrm{kpc}$ and $30 \mathrm{~km} \mathrm{~s}^{-1}$ ) will enable detection of populations comparable to those determined for the Local Group, implying increased numbers by some two orders of magnitude. If these low mass populations are not found, then the Local Group hypothesis for the CHVCs must be seriously reconsidered.

Acknowledgements. We are grateful to Martin Zwaan for providing details of the statistical analysis presented by Zwaan and Briggs (2000). We acknowledge extensive use of the LEDA database, www-obs.univ-lyon1.fr. This research has made use of the NASA/IPAC Extragalactic Database (NED) which is operated by the Jet Propulsion Laboratory, California Institute of Technology, under contract with the National Aeronautics and Space Administration.

\section{References}

Banks, G. D., Disney, M. J., Knezek, P. M., et al. 1999, ApJ, 524,612

Blitz, L., Spergel, D. N., Teuben, P. J., Hartmann, D., \& Burton, W. B. 1999, ApJ, 514, 818

Braun, R., \& Walterbos, R. A. M. 1992, ApJ, 386, 120

Braun, R., \& Burton, W. B. 1999, A\&A, 341, 437

Braun, R., \& Burton, W. B. 2000, A\&A, 354, 853

Burton, W. B., Braun, R., \& Chengalur, J. N. 2001, A\&A, 369, 616

Courteau, S., \& van den Bergh, S. 1999, AJ, 118, 337

Deul, E. R., \& van der Hulst, J. M. 1987, A\&AS, 67, 509

Garcia, A. M. 1993, A\&AS, 100, 47

Hartmann, D., \& Burton, W. B. 1997, Atlas of Galactic Neutral Hydrogen (Cambridge: Cambridge University Press)

Kamphuis, J. 1993, Ph.D. Thesis, Univ. Groningen

Kamphuis, J., \& Briggs, F. H. 1992, A\&A, 253, 335

Kilborn, V. A., Staveley-Smith, L., Marquarding, M., et al. 2000, AJ, 120, 1342

Klypin, A., Kravtsov, A. V., Valenzuela, O., \& Prada, F. 1999, ApJ, 522, 82

Lo, K. Y., \& Sargent, W. L. W. 1979, ApJ, 227, 756

Luks, T., \& Rohlfs, K. 1992, A\&A, 263, 41

Mulchaey, J. S., Davis, D. S., Mushotzky, R. F., \& Burstein, D. 1996, ApJ, 456, 80 
Pisano, D. J., \& Wilcots, E. M. 1999, AJ, 117, 2168

Pisano, D. J., \& Wilcots, E. M. 2000, in Gas \& Galaxy Formation, ed. Hibbard, Rupen, \& Van Gorkom, ASP Conf. Ser.

Sandage, A. 1986, ApJ, 307, 1

Sorar, E. 1994, Ph.D. Thesis, Univ. Pittsburgh

Spitzak, J. G., \& Schneider, S. E. 1998, ApJS, 119, 159

Sandage, A. 1986, ApJ, 307, 1

Taylor, C. L., Brinks, E., Grashuis, R. M., \& Skillman, E. D. 1995, ApJS, 99, 427; erratum 102, 189 (1996)

Taylor, C. L., Thomas, D. L., Brinks, E., \& Skillman, E. D. 1996, ApJS, 107, 143 van Gorkom, J. H., Bahcall, J. N., Jannuzi, B. T., \& Schneider, D. P., AJ, 106, 2213

van Woerden, H., Schwarz, U. J., Peletier, R. F., Wakker, B. P., \& Kalberla, P. M. W. 1999, Nature, 400, 138

Wakker, B. P., et al. 1999, Nature, 402, 388

Wakker, B. P., \& van Woerden, H. 1991, A\&A, 250, 509

Wolfire, M. G., McKee, C. F., Hollenbach, D., \& Tielens, A. G. G. M. 1995, ApJ, 453, 673

Zwaan, M. A., Briggs, F. H., Sprayberry, D., \& Sorar, E. 1997, ApJ, 490, 173

Zwaan, M. A., \& Briggs, F. H. 2000, ApJ, 530, L61

Zwaan, M. A. 2001, MNRAS, in press [astro-ph/0103328] 